EDITORIAL REVIEW ARTICLE

\title{
Sleep Related Breathing Disorders in Interstitial Lung Diseases
}

\section{J. M. Joshi}

Department of Respiratory Medicine, T. N. Medical College and B. Y. L. Nair Hospital, Mumbai

Keywords: Sleep disordered breathing (SDB), sleep apnea syndromes, idiopathic pulmonary fibrosis (IPF)

Indian J Sleep Med 2007; 2.1, 1-4

$\mathrm{T}$

he interstitial lung diseases (ILDs) are a heterogeneous group of disorders that present with similar signs and symptoms. The term idiopathic interstitial pneumonia (IIP) refers to a subgroup of interstitial lung diseases without known cause. (1) Idiopathic pulmonary fibrosis (IPF) or cryptogenic fibrosing alveolitis (CFA) is the most common of the various IIPs constituting more than half the cases. (2) Normal sleep is associated with changes in respiration and the impact of sleep on patients with underlying lung disease may be significant. Some recent studies have attempted to assess the prevalence of abnormal sleep structure, oxygenation and prevalence of sleep related breathing disorders (SRBDs) in patients with ILDs. In the absence of effective treatments for IPF, it is important to diagnose and treat co morbid conditions like (SRBDs), which may lead to improvements in quality of life.

Three patterns of breathing disorders during sleep have been reported in patients with ILDs, i) oxygen desaturation occurring primarily during rapid eye movement (REM) sleep, ii) a sustained fall in saturation during both non-REM (NREM) and REM sleep and iii) snorers, some of whom have the classic obstructive sleep apnea (OSA). $(3,4,5,6)$ OSA has been described in patients with other disorders

Address for correspondence:

Dr J. M. Joshi

Professor and Head

Department of Respiratory Medicine,

T. N. Medical College and B. Y. L. Nair Hospital, Mumbai-400008; Tel: 02223081490 Extn -642, 643;

Fax : 02223003095

Email: drjoshijm@email.com,

deptrespmed@hathway.com that may cause ILDs, including rheumatoid arthritis (7) and sarcoidosis. (8) Nocturnal oxygen therapy is recommended for ILDs with nocturnal hypoxemia, while use of continuous positive airway pressure (CPAP) is the now established treatment for OSA.

In a recent study published from Cleveland Clinic Sleep Disorders Center, United States, all-night polysomnogram (PSG) was performed from 2001 to 2005 , to investigate complaints suggestive of sleep-disordered breathing in 18 of 857 IPF patients. (9) All patients showed a reduction in sleep efficiency, REM sleep, and slow wave sleep. OSA was confirmed in 11 of the 18 patients with complaints suggestive of sleep apnea, while the remaining 7 patients were diagnosed as primary snoring or upper airway resistance syndrome (UARS). The overall apnea-hypopnoea index (AHI) and REM AHI were negatively correlated with forced expiratory volume in 1 second FEV (1) indicating that significant impairment in pulmonary function testing may be associated with OSA in cases of IPF. The AHI correlated positively with body mass index and the authors suggested that an increased BMI might be a predictor of OSA in these cases. A limitation of this study was that only patients having symptoms of sleep-disordered breathing were studied. However, in another recent study (10) PSG was performed in all patients with interstitial lung disease. PSG results showed that, patients had overall poor sleep efficiency, they spent more time as wake after sleep onset (WASO) and total sleep time, time spent in NREM sleep stage III and IV, and in REM sleep was decreased. OSA was diagnosed in 24 of 37 cases $(64.9 \%)$. No difference was found in the BMI between the patients with and without OSA. It can therefore be concluded that obesity

Indian Journal of Sleep Medicine (IJSM), Vol. 2, No. 1, 2007 
may not be the only predisposing factor for OSA in cases of ILDs.

Hypoxemia is likely to be a critical factor in pathogenesis of (SRBDs). McNicholas et al (11) have suggested that hypoxia induced brainstem depression may be the mechanism for development of breathing disorders in sleep. Patients with ILDs have a rapid shallow breathing pattern while awake that is thought to be due to activation of lung reflexes, while sleep results in changes in respiratory control and causes hypoxemia. Oxygen saturation during sleep drops in patients of ILDs, more during REM sleep and especially in those with more severe awake hypoxemia. (5) Aydogdu M, et al in their recent study (10) have confirmed severe oxygen desaturations during sleep in cases of ILD and there was a statistically significant positive correlation between mean awake $\mathrm{O} 2$ saturation and mean and lowest sleep $\mathrm{O} 2$ saturations. They also showed statistically significant negative correlations between Clinical, Radiological and Physiological (CRP) scoring system used to assess the disease stage and awake sleep $\mathrm{O} 2$ saturations. Patients with $\mathrm{SaO}_{2}$ less than $90 \%$ are reported to have more disrupted sleep than those with $\mathrm{SaO}_{2}$ above $90 \%$. (12) Low oxygen saturation during sleep in ILD is related to awake $\mathrm{PaO} 2$, age and lung compliance, predicted by the equation: Predicted $\mathrm{SaO} 2=75+0.23(\mathrm{PaO} 2)-0.2$ (age) (13) Another recent study (14) confirmed that daytime oxygen level predicted mean overnight $\mathrm{SaO}_{2}$ but percentage predicted forced vital capacity (FVC) did not. Thus, patients of ILDs with daytime $\mathrm{Sa}_{\mathrm{O} 2}$ of less than $90 \%$ and/or a history of snoring during sleep are likely to develop sleep disturbances. These patients tend to have reduced rapid eye movement (REM) sleep, lighter and more fragmented sleep, hypoxemia during REM sleep and sleep disordered breathing. $(2,9,10)$

One study (14), performed to investigate the impact of oxygen on sleep and breathing in patients with ILD in Mexico City, at 2,240 m of altitude could not demonstrate similar effect of hypoxia on sleep efficiency and arousal index in the patients of ILD acclimatized to the moderate altitude. Despite the severe hypoxemia found in these patients during sleep, the quality of sleep was not significantly different from healthy controls and it did not change significantly with oxygen. However, the authors suggested that differences in the populations, type of interstitial lung disease, PSG equipment and environment may account in part for the discrepancy found in the sleep quality between their patients and previous reports $(11,12)$ including subsequent studies $(9,10)$.

Arterial $\mathrm{PaCO}_{2}$ is another factor, which has a dominant influence on breathing. A carefully performed study (16) done during sleep in normal humans showed that central apnea may be induced when the $\mathrm{PaCO}_{2}$ is experimentally lowered by 1 to $3 \mathrm{~mm} \mathrm{Hg}$ below the resting $\mathrm{PaCO}_{2}$ while patients are awake. Data on patients with heart failure also show that a low $\mathrm{PaCO}_{2}$ results in apneas during sleep. (17) Patients with ILDs characteristically exhibit increased ventilation and breathing frequency when awake and these increases persist during deep nonREM sleep. (4) It appears that patients of ILDs with tachypnoea while awake maintain their tachypnoea during sleep and this maintenance of rapid breathing during sleep suggests that the reflexes causing the rapid shallow breathing were active during the sleep phase as well. (2) The resultant level of ventilation is usually excessive for the level of carbon dioxide $(\mathrm{CO} 2)$ production leading to hypocapnea, (18) which may predispose to ventilatory instability and sleep related breathing disorders in patients with ILDs.

In a study published in an earlier issue of this journal (19) we studied 12 patients of ILDs with OSA diagnosed by night-time recording of respiratory variables (limited PSG). None of the patients had complaints of snoring or excessive daytime sleepiness. The mean AHI was $23.33(+/$ - 6.27), which showed significant improvement $(\mathrm{p}<0.001)$ in the AHI to $8(+/-8.03)$ on breathing 2 liters per minute of supplemental oxygen. Ten out of the 12 patients had a BMI of less then $25 \mathrm{Kg} / \mathrm{m}^{2}$, while 2 patients had a BMI of $27 \mathrm{Kg} / \mathrm{m}^{2}$. All patients had restrictive abnormality on spirometry and the awake mean partial pressure of oxygen was $71.88(+/-12.44)$. None except one patient was hypercapnic mean daytime partial pressure of carbon dioxide was $36.39(+/-8.27)$. We showed that nocturnal oxygen therapy not only had a beneficial effect on oxyhaemoglobin saturation but on the obstructive events as well.

Oxygen supplementation has been believed to have a deleterious effect on OSA by prolonging the apneas and hypopnoeas perhaps by the removal of hypoxic ventilatory drive. (20) Gold et al (21) found a reduction in central and mixed apnea with nasal oxygen in nine patients but the frequency of obstructive apneas doubled. However, Breitenbucher et al (22) studied the effect of nocturnal lowflow oxygen administration in 10 consecutive patients with obstructive sleep apnea and in 5 patients the clinical 
symptoms disappeared completely with nasal oxygen administration. In the remaining 4 of 5 patients, insertion of a transtracheal catheter for nocturnal oxygen delivery resulted in a favorable outcome. Both central and obstructive apneas may result, depending upon the propensity of the upper airways to collapse, when cyclical output to respiratory muscles occurs during sleep (23) in response to the stimuli (i.e. hypoxia or hypocapnea). The mechanism of apnea elimination by nasal oxygen could be either by relieving the hypoxic brain stem suppression or by restoring a normal breathing pattern in hypocapneic patients by reducing the hypoxic influence on ventilation. (24) However, as shown in the study by Vazquez and Perez-Padilla (15) although oxygen reduced heart rate and breathing frequency in ILD patients during sleep, it did not normalize the breathing frequency. It is possible that oxygen works by correction of dysfunction of the chemoreflex mechanism (ventilatory responses to chemical stimuli), which is secondary to repeated severe episodes of nocturnal hypoxemia in patients with ILD. (5)

Identification and correction of the sleep disturbance and the use of supplemental oxygen during sleep has been recommended in IPF and perhaps other ILDs because this may reduce morbidity and improve patient survival, especially regarding the pulmonary hypertension and cor pulmonale that develop in these patients. (2) The present data suggests that patients of ILDs may have sleep disordered breathing in addition to nocturnal desturations. The severity of ventilatory defect, BMI and daytime oxygenation are likely predictors of SRDBs in these cases. The diagnosis of OSA in cases with ILDs would require careful consideration regarding treatment options, nocturnal oxygen desaturation requiring supplemental oxygen and OSA requiring CPAP therapy. However, oxygen supplementation may be effective in reversing both apneas and oxygen desaturation at least a subgroup of cases. Chronic Obstructive Pulmonary Disease (COPD) with OSA is called the "Overlap Syndrome" and is explained by high prevalence of the two disorders, similar risk factors; male gender, age, cigarette smoking and control of breathing defect predisposing to OSA. The same could be applicable to ILDs and OSA. Larger studies are required to study the prevalence of sleep disordered breathing, to determine whether sleep study/ polysomnography (PSG) should be considered as part of the assessment in patients with ILDs and the optimum treatment for "the overlap of ILD and OSA."

\section{References}

1. Chapman JT, Farver CF. Idiopathic Interstitial Lung Disease: A Review of Recent Classifications. Interstitial, Inflammatory, and Occupational Lung Disease Clinical Pulmonary Medicine. 2004. 11:17-24.

2. American Thoracic Society. Idiopathic pulmonary fibrosis: diagnosis and treatment. International consensus statement. American Thoracic Society (ATS) and the European Respiratory Society (ERS). Am J Respir Crit Care Med. 161; 2000: 646-664.

3. Bye PT, Issa F, Berthon-Jones M, Sullivan CE. Studies of oxygenation during sleep in patients with interstitial lung disease. Am Rev Respir Dis. 1984; 129:27-32.

4. Shea SA, Winning AJ, McKenzie E, Guz A. Does the abnormal pattern of breathing in patients with interstitial lung disease persist in deep, non-rapid eye movement sleep? Am Rev Respir Dis. 1989 ; 139:653-8.

5. Tatsumi K, Kimura H, Kunitomo F, et al. Arterial oxygen desaturation during sleep in interstitial pulmonary disease. Correlation with chemical control of breathing during wakefulness. Chest 1989;95;962-967.

6. Hira HS, Sharma RK. Study of oxygen saturation, breathing pattern and arrhythmias in patients of interstitial lung disease during sleep. Indian J Chest Dis Allied Sci. 1997;39: 157-62

7. Offers E, Herbort C, Dumke K, et al. Complicated course of rheumatoid arthritis with pulmonary involvement, myocardial fibrosis and sleep apnea syndrome. Pneumologie. 1996; 50:906-911.

8. Turner GA, Lower EE, Corser BC, et al. Sleep apnea in sarcoidosis. Sarcoidosis Vasc Diffuse Lung Dis. 1997; 14:61-64

9. Charalampos Mermigkis, Jeffrey Chapman, Joseph Golish, et al. Sleep-Related Breathing Disorders in Patients with Idiopathic Pulmonary Fibrosis. Lung. 2007 Apr 10; [Epub ahead of print] DOI: 10.1007/s00408-007-9004-

10. Aydogdu M, Ciftci B, Firat Guven S, et al. Assessment of sleep with polysomnography in patients with interstitial lung disease. Tuberk Toraks. 2006;54:213-21.

11. McNicholas WT, Carter JL, Rutherford R, Zamel J, Phillipson EA. Beneficial effect of oxygen in primary alveolar hypoventilation with central sleep apnea. Am Rev Respir Dis 1982; 125: 773-775.

12. Perez Padilla $\mathbf{R}$, West $P$, Lertzman $M$ et al. Breathing during sleep in patients with interstitial lung disease. Am Rev Respir Dis $1985 ; 132: 224-229$

13. Rajagopal KR, Derderian SS, Culpepper WJ, Et al. Sleep and nocturnal oxygenation in patients with restrictive pulmonary diseases. Sleep Res 1991; 20A: 445.

14. Clark M, Cooper B, Singh S,et al. A survey of nocturnal hypoxaemia and health related quality of life in patients with cryptogenic fibrosing alveolitis. Thorax. 2001;56:4826.

15. Vazquez JC, Perez-Padilla R. Effect of oxygen on sleep and 
breathing in patients with interstitial lung disease at moderate altitude. Respiration. 2001;68:584-9.

16. Javaheri S, Corbett BS. Association of Low $\mathrm{PaCO}_{2}$ with Central Sleep Apnea and Ventricular Arrhythmias in Ambulatory Patients with Stable Heart Failure. Ann Intern Med 1998; 128:204-207.

17. Skatrud JB, Dempsey JA. Interaction of sleep state and chemical stimuli in sustaining rhythmic ventilation. J Appl Physiol. 1983; 55:813-22.

18. Lourenco RV, Turino GM, Davidson LA, et al. The regulation of ventilation in diffuse pulmonary fibrosis. Am J Med. 1965;38: 199-216.

19. Shah DV, Joshi JM. Role of nocturnal oxygen therapy in interstitial lung disease with obstructive sleep apnea syndrome. Indian J Sleep Med 2006; 1. 1: 41-44.

20. Motta J, Guilleminault C. Effects of oxygen administered in sleep induced apneas. In: Guilleminault C, Dement W,
Eds. Sleep Apnea Syndromes. New York, NY: Alan \& Liss; 1978: 137-144.

21. Gold AR, Bleeker ER, Smith PL. Shifts from central and mixed sleep apnea to obstructive apnea resulting from low flow oxygen. Am Rev Respir Dis 1985; 132: 220-223.

22. Breitenbucher A, Keller-Wossidlo H, Keller R. Transtracheal oxygen therapy in obstructive sleep apnea syndrome. Schweiz Med Wochenschr. 1989; 1 19:1638-41.

23. White DP. Central Sleep Apnea. In WB Saunders Company. Kryger MH, Roth T, Dement W, 3 rd ed. Principles and Practice of Sleep Medicine. Philadelphia 2000: 827-839.

24. Smith PL, Haponik EF, Bleecker ER. The effects of oxygen in patients with sleep apnea. Am Rev Respir Dis. 1984;130:958 63

25. Meeker DP. Sleep in the patient with lung disease. Cleve Clin J Med. 1992:59:157-65 